\title{
How evidence-based is dentistry anyway? From evidence-based dentistry to evidence-based practice
}

\author{
Shaun Sellars ${ }^{1}$
}

\section{Key points}

The majority of dentists are aware of evidencebased dentistry, but don't necessarily apply it in practice.
Barriers to implementation of evidence-based dentistry include time, inadequate training and lack of relevance to practice.
Uptake of evidence in practice could be improved by production of evidence-based clinical practice guidelines.

\begin{abstract}
Since its beginnings in the mid-1990s, evidence-based dentistry (EBD) has gradually gained acceptance into the understanding of dental practitioners. Despite this, most treatment provided by dentists cannot truly be described as being based on experimental evidence. The aim of this paper is to explore the reasons why EBD has not yet reached its full potential. The weakness of evidence produced, as well as the attitude of dentists towards EBD, will be examined. The role of the trusted source in disseminating information will be discussed and examples are given of how this concept could be used to further integrate evidence into everyday practice. Suggestions will be made regarding how research waste can be cut by producing less but more relevant research. Furthermore, realistic ideas on how EBD can be utilised in practice will be provided with the aim to shift towards the concept of evidence-based practice (EBP).
\end{abstract}

\section{Introduction}

Archie Cochrane kick-started the evidencebased medicine machine into action with the publication of Effectiveness and Efficiency: Random Reflections of Health Services in 1971. ${ }^{1}$ By 1992, 'evidence-based medicine' was formally recognised as a way of teaching medicine. ${ }^{2}$ Dentistry soon followed suit, with 'evidence-based dentistry' (EBD) first being coined by Richards and Lawrence in 1995. ${ }^{3}$ Since then, EBD has been clearly defined as the combination of a dentist's clinical expertise, the needs and preferences of patients, and the use of the most recent and clinically relevant evidence to provide an improved standard of care.4

Despite this, it has been shown that many dentists pay only lip service to EBD and employ it vaguely. If we are to improve upon this, then we need to move towards producing evidence that dentists can easily apply to their everyday

${ }^{1} G e n e r a l$ Dentist, Alvington House Dental Practice, 112 Northgate Street, Bury St Edmunds, IP33 1HP, UK Correspondence to: Shaun Sellars

Email address: s.sellars@alvington-house.co.uk

Refereed Paper

Accepted 9 April 2020

https://doi.org/10.1038/s41415-020-1785-2 practice. This shift will propel dentistry into the realm of evidence-based practice (EBP); that is, practice that uses realistic, evidencebased approaches to diverse clinical problems. EBP provides us with up-to-date approaches to patient care which are scientific, safe, efficient and cost-effective. ${ }^{6}$

While Archie Cochrane advocated the use of randomised controlled trials (RCTs) to formulate the best quality of evidence, we will see that this is not always practicable or even desirable in dentistry. ${ }^{7,8}$ Alternative ways in which evidence can be collected and delivered to dentists need to be sought to maximise integration into practice and benefit to patients.

\section{Evidence base}

When EBD was first conceived, it was thought that dentists were uninterested in research papers. ${ }^{9}$ Now, there are hundreds of journals producing thousands of research papers every year. ${ }^{10}$

Dentists have been slow on the uptake of evidence-based techniques. ${ }^{10,11,12}$ It may be the case that there is simply too much information out there for dentists to filter through and apply to practice. The information overload makes it difficult for dentists to ascertain which research is worthwhile of their attention. No single practitioner is able to appraise the evidence available. It has been estimated that, for dentists to base their care on the current highest quality evidence, they would need to read and evaluate at least one paper per day for the rest of their practising lives. ${ }^{13}$

While the amount of evidence that is available has increased, there are still issues with the quality of that evidence. The highest quality of research-based evidence is the product of the systematic review. These reviews assimilate and summarise the results of other studies to produce high-level evidence for interventions. However, the number of systematic reviews relevant to clinical dentistry has traditionally been low (although this is improving). ${ }^{10}$ Systematic reviews are only useful if they provide answers to clinical questions. Out of the 170 Cochrane systematic reviews on dentistry and oral health, the vast majority are unable to provide robust clinical recommendations. This is due to there either being a lack of research or the research that exists generally being of low quality. Systematic reviews are also unable to keep up with the pace of development of new dental materials and techniques.

As with any form of research, there exists 
the risk of bias, especially publication bias. Publication bias exists because, traditionally, only studies which show positive or significant results are likely to get published. Those with negative results, or with results that are not statistically significant, often remain unpublished or are published after some delay, and often in journals with lower impact factors. ${ }^{14}$

\section{Uptake of evidence by dentists}

The majority of dentists are aware of the existence of $\mathrm{EBD}^{12,15,16,17}$ and that dentists generally have a positive attitude to the provision of EBD. ${ }^{16}$ So why are dentists not putting EBD into practice?

The main barriers to the implementation of EBD into practice have been identified in multiple studies. These are generally reported to be due to insufficient time, inadequate training in EBD and lack of relevance of the evidence to their practice. ${ }^{16,17,18}$ In addition to this, it has repeatedly been shown that when dentists are searching for help or advice, they would instead turn to colleagues or textbooks than research papers. ${ }^{3,11,17,18}$ The tangible evidence of a clinician's eyes and hands is trusted above that of the abstract evidence provided by research papers. ${ }^{18}$ This is compounded by the inability of dentists to access primary evidence due to a significant amount being held behind paywalls.

It is clear that there needs to be a shift in emphasis away from merely providing evidence for dentists to sieve through. Easyto-implement solutions need to be provided, which lead to simple and evidence-based improvements to everyday practice. There needs to be a refocusing from EBD to EBP.

\section{Moving towards EBP}

Dentistry, as with other forms of healthcare, has traditionally been grounded in the opinions and experience of experts and peers. Utilising evidence from research flies in the face of this traditionalistic approach and can expect to be resisted. One of the common misunderstandings of EBD is that it ignores clinical experience. ${ }^{17}$ On the contrary, EBD explicitly incorporates the use of clinical judgement as well as patient desires when making treatment decisions.

One of the weaknesses of $\mathrm{EBD}$, as it is currently understood, is that it does not explicitly factor in real-world practising issues (such as cost and ease of implementation of techniques). This lack of perceived real-world application is a factor affecting uptake of EBD in practice. ${ }^{18}$ Here is where the shift to EBP comes into its own, incorporating best evidence for interventions with the clinical experience of practitioners, patient desires and practice-based factors. When allowing for these substantive issues in delivering EBD, EBP could, in theory, be more easily taken up by practising dentists. Formulating easyto-follow guidelines to implement evidencebased interventions into routine care would also help eliminate the time barrier that exists in integrating evidence into practice.

Moving towards EBP has a multitude of benefits for practitioners, patients and practice teams. From a practitioner point of view, EBP gives increased confidence in decisionmaking ability and security in knowing that the treatment provided maximises benefits and reduces harm. Patients are increasingly able to place their trust in their dentists and dental teams are more motivated in their jobs. ${ }^{6}$

\section{Evidence-based research}

Although Archie Cochrane is rightly heralded as the founder of the evidence-based healthcare movement, it could be argued that his reliance on RCTs to direct healthcare interventions has now begun to stifle the uptake of evidence in practice. ${ }^{19}$ Not all clinical problems in dentistry can be answered by RCTs for reasons of ethics and feasibility. ${ }^{78}$ It is also clear that when RCTs are carried out, the research question is not always tailored to provide a beneficial clinical outcome.

To improve the quality of research, there needs to be a focus on producing less research but of higher quality, which answers questions relevant to clinical practice. ${ }^{20}$ The first stage of this would be to improve the formulation of research questions to make the answers more clinically relevant. The obvious way to do this would be to integrate qualitative research methods into dental research further.

Qualitative research methods, such as interviews and focus groups, answer the questions such as 'how' and 'why' as opposed to 'how many' or 'how often.' Rather than giving numerical results, these methods elicit a rich dataset, giving a depth of understanding that cannot be measured with purely quantitative research.

The effect of this would be twofold. Initially, qualitative methods could be utilised to improve the quality of research questions being asked in quantitative research. Qualitative research is ideal for discovering what kind of questions need to be asked to ensure maximum uptake of the results of RCTs and systematic reviews, making the subsequent quantitative research more appropriate and reducing research waste.

Secondly, qualitative research methods have a place as research methods in their own right. For example, it is impossible to give a numerical answer to the question: 'What interventions are most effective at changing the way in which dentists practise?' It is here, away from the cold hard statistics of the RCT, where qualitative research shines. It can also help give a depth of understanding when trying to make sense of the results that RCTs have given.

Finally, standardisation of reporting data will enable the production of research that can be assimilated into systematic reviews. ${ }^{21}$

\section{Trusted sources}

It is not feasible for a dentist to read and appraise every paper on every clinical topic they come across. Dentists prefer to turn to colleagues, key opinion leaders, company sales representatives or social media forums to gain new information. These trusted sources are not guaranteed to be up-to-date or even correct. Key opinion leaders and company representatives are likely to provide financially motivated advice. Social media forums have no practical way of exerting a quality control over what is posted, and yet due to the visibility of cases posted, they are likely to have an overinflated effect on practice. This is despite the lack of control over case presentations and information distribution. Dentists need to be aware of the pitfalls of following techniques espoused on such platforms unless there is evidence to back them up.

One answer to these problems is to utilise this reliance on the trusted source and develop easy-to-access, easy-to-follow, evidencebased clinical practice guidelines (CPGs). ${ }^{6}$ These CPGs can be formally structured, incorporating high-quality evidence, but allow the values of patients and practitioners to be expressed. These guidelines could, in turn, aid each practitioner to develop their own standard operating procedure for elements of their practice.

The concept of a dental reader has been introduced previously, ${ }^{22}$ and there are already such guidelines readily available from 
the Scottish Dental Clinical Effectiveness Programme (SDCEP) ${ }^{23}$ and the Faculty of General Dental Practice (FGDP) UK. ${ }^{24}$ The SDCEP guidelines, in particular, are easy to access, both online and via mobile phone app, and have been well accepted across the profession.

The extent of these guidelines, however, is limited, and in order to progress to a more evidence-based profession, further funding and input is required to expand into other clinical and non-clinical areas.

\section{Conclusion}

Evidence-based healthcare has come a long way in the decades since its inception. There is now more evidence being produced than practitioners can reasonably be expected to filter through and apply to their practice. This problem is exacerbated by the amount of wasted research data generated due to research questions not being tailored to clinical outcomes.

In addition to this, dentists have been slow to uptake the ideas of EBD due to a perceived lack of time or understanding. To improve this uptake of evidence and move towards a more evidence-based way of practising dentistry, it is suggested that qualitative research methods are more fully integrated into the research lexicon. This would allow the formulation of more suitable research questions on which to base quantitative research, as well as enable a deeper understanding of research already conducted and answer non-clinical questions.

The way that we assess evidence needs to change. Best available evidence must be cumulative, taking a Bayesian stance to information gathering. It also needs to be patient-centred while focusing on costeffectiveness and benefit. This may mean that research data may not be optimal, but take a pragmatic approach, ensuring it is applicable to a practice setting. ${ }^{25}$

Dentists are often unable or unwilling to access primary evidence. Efforts should, therefore, be made to promote and improve access to summaries of evidence. ${ }^{26}$ The move towards truly EBP is only likely to occur with the further development of easy-to-follow CPGs, as already produced by SDCEP and FGDP(UK). This would be aided by the introduction of the role of 'dental reader' to assimilate the already existing information and identify areas where gaps in the evidence exist. In this way, the concept of the 'trusted source' can be utilised to the good of the profession.

How evidence-based is dentistry anyway? To paraphrase physician and World Health Organisation statistician Hans Rosling, it is 'not great, but getting better.'27

\section{References}

1. Cochrane A L. Effectiveness and Efficiency: random reflections on health services. London: Nuffield Provincial Hospitals Trust, 1972. Available online at https:// www.nuffieldtrust.org.uk/research/effectivenessand-efficiency-random-reflections-on-health-services (accessed June 2020).

2. Evidence-Based Medicine Working Group. EvidenceBased Medicine: A New Approach to Teaching the Practice of Medicine. JAMA 1992; 268: 2420-2425.

3. Richards $\mathrm{D}$, Lawrence $\mathrm{A}$. Evidence-based dentistry. $\mathrm{Br}$ Dent J 1995; 179: 270-273.

4. ADA Center for Evidence-Based Dentistry. About EBD. 2020. Available at https://ebd.ada.org/en/about (accessed March 2020)

5. Nieri M, Mauro S. Continuing Professional Development of Dental Practitioners in Prato, Italy. J Dent Educ 2008; 72: 616-625.

6. Kishore M, Panat SR, Aggarwal A, Agarwal N, Upadhyay N, Alok A. Evidence-based dental care: integrating clinical expertise with systematic research. IClin Diagn Res 2014; 8: 259-262.

7. Martin D. Evidence-based dentistry: let's talk about experimental evidence. Br Dent J 2019; 226: 557-558.

8. Roulet J-F. A consensus-based approach to evidencebased clinical practice. Dent Mater 2017; 33: 1067-1068.

9. Grace M. Evidence-based dentistry. Br Dent J 2002; 193: 545 .
10. Kao R T. The Challenges of Transferring Evidence Based Dentistry into Practice. J Evid Based Dent Pract 2006; 6: 125-128.

11. Yamalik N, Nemli S K, Carrilho E et al. Implementation of evidence-based dentistry into practice: analysis of awareness, perceptions and attitudes of dentists in the World Dental Federation - European Regional Organisation Zone. Int Dent J 2015; 65: 127-145.

12. Al-Ansari A, ElTantawi M. Factors Affecting SelfReported Implementation of Evidence-Based Practice Among a Group of Dentists. J Evid Based Dent Pract 2014; 14: 2-8.

13. Niederman R. Guest editorial: Evidence-based dentistry finds a new forum Exelauno. J Am Dent Assoc 2009. 140: 272-274.

14. Papageorgiou S N, Dimitraki D, Coolidge T, Kotsanos N. Publication Bias \& Small-study Effects in Paediatric Dentistry Meta-analyses. J Evid Based Dent Pract 2015; 15: 8-24.

15. Yus of Z Y M, Han L J, San P P, Ramli A S. EvidenceBased Practice Among a Group of Malaysian Dental Practitioners. J Dent Educ 2008; 72: 1333-1342.

16. Straub-Morarend C L, Marshall TA, Holmes D C, Finkelstein M W. Toward Defining Dentists' EvidenceBased Practice: Influence of Decade of Dental School Graduation and Scope of Practice on Implementation and Perceived Obstacles. J Dent Educ 2013; 77: 137-145.

17. Iqbal A, Glenny A-M. General dental practitioners' knowledge of and attitudes towards evidence based practice. Br Dent J 2002; 193: 587-591.

18. Sbaraini A, Carter S M, Evans R W. How do dentists understand evidence and adopt it in practice? Health Educ J 2012; 71: 195-204

19. Fox C, Kay E J, Anderson R. Evidence-based dentistry - overcoming the challenges for the UK's dental practitioners. Br Dent J 2014; 217: 191-194.

20. Clarkson J, Worthington H. Leadership in evidence based dentistry. J Dent 2019; 87: 16-19.

21. Lang L A, Teich S T. A critical appraisal of evidencebased dentistry: The best available evidence. J Prosthet Dent 2014; 111: 485-492.

22. Sellars $\mathrm{S}$, Wassif $\mathrm{H} \mathrm{S}$. Is dentistry the orphaned field of medicine? Ethical consideration for evidence-based dentistry. Br Dent J 2019; 226: 177-179.

23. Scottish Dental Clinical Effectiveness Programme. Published Guidance. 2020. Available online at http:// www.sdcep.org.uk/published-guidance/ (accessed March 2020).

24. Faculty of General Dental Practice (UK). Guidance \& Standards. 2020. Available online at https://www.fgdp org.uk/guidance-standards (accessed March 2020).

25. Chiappelli F. Evidence-Based Dentistry: Two Decades and Beyond. J Evid Based Dent Pract 2019; 19: 7-16.

26. McColl A, Smith H, White P, Field J. General practitioners' perceptions of the route to evidence based medicine: a questionnaire survey. BMJ 1998; 316: 361-365.

27. Rosling H, Rosling O, Rönnlund A R. Factfulness: Ten Reasons We're Wrong About The World - And Why Things Are Better Than You Think. London: Sceptre, 2018. 